\title{
PARACOMPACTNESS AND PRODUCT SPACES
}

A. H. STONE

A topological space is called paracompact (see [2]) ${ }^{1}$ if (i) it is a Hausdorff space (satisfying the $T_{2}$ axiom of [1]), and (ii) every open covering of it can be refined by one which is "locally finite" (= neighbourhood-finite; that is, every point of the space has a neighbourhood meeting only a finite number of sets of the refining covering). J. Dieudonné has proved [2, Theorem 4] that every separable metric (=metrisable) space is paracompact, and has conjectured that this remains true without separability. We shall show that this is indeed the case. In fact, more is true: paracompactness is identical with the property of "full normality" introduced by J. W. Tukey [5, p. 53]. After proving this (Theorems 1 and 2 below) we apply Theorem 1 to obtain a necessary and sufficient condition for the topological product of uncountably many metric spaces to be normal (Theorem 4).

For any open covering $\mathscr{W}=\left\{W_{\alpha}\right\}$ of a topological space, the star $(x, W)$ of a point $x$ is defined to be the union of all the sets $W_{\alpha}$ which contain $x$. The space is fully normal if every open covering $V$ of it has a " $\Delta$-refinement" $W$-that is, an open covering for which the stars $(x, \mathscr{W})$ form a covering which refines $U$.

THeOREM 1. Every fully normal $T_{1}$ space is paracompact.

Let $S$ be such a space, and let $V=\left\{U_{\alpha}\right\}$ be a given open covering of $S$. (We must construct a locally finite refinement of $U$. Note that $S$ is normal $\left[5\right.$, p. 49] and thus satisfies the $\mathrm{T}_{2}$ axiom.)

There exists an open covering $U^{1}=\left\{U^{1}\right\}$ which $\Delta$-refines $V$, and by induction we obtain open coverings $V^{n}=\left\{U^{n}\right\}$ of $S$ such that $U^{n+1} \Delta$-refines $U^{n}(n=1,2, \cdots$, to $\infty)$. For brevity we write, for any $X \subset S$,

$$
\begin{aligned}
(X, n) & =\text { star of } X \text { in } U^{n} \\
& =\text { union of all sets } U^{n} \text { meeting } X
\end{aligned}
$$

(roughly corresponding to the " $1 / 2^{n}$-neighbourhood of $X$ " in a metric space), and

$$
(X,-n)=S-(S-X, n) .
$$

Presented to the Society, October 25, 1947; received by the editors October 25, 1947.

${ }^{1}$ Numbers in brackets refer to the bibliography at the end of the paper. 
Thus, since the set $(X, n)$ is evidently open, $(X,-n)$ is closed. Further, it is easily seen that

$$
(X,-n)=\{x \mid(x, n) \subset X\},
$$

where $(x, n)$, in conformity with (1), denotes the star of $x$ in $\mho^{n}$; and it readily follows that

$$
((X,-n), n) \subset X
$$

From the definition of $\Delta$-refinement we have

$$
((X, n+1), n+1) \subset(X, n) .
$$

The trivial relations $X \subset Y \rightarrow(X, n) \subset(Y, n), m \geqq n \rightarrow(X, m)$ $C(X, n), \bar{X} \subset(X, n)$, and $y \in(x, n) \leftrightarrow x \in(y, n)$ will also be useful.

For convenience, suppose the sets $U_{\alpha}$ of $U$ are well-ordered.

Now define, for each $\alpha$,

$$
V_{\alpha}^{1}=\left(U_{\alpha},-1\right), V_{\alpha}^{2}=\left(V_{\alpha}^{1}, 2\right), \text { and } V_{\alpha}^{n}=\left(V_{\alpha}^{n-1}, n\right) \quad(n \geqq 2) .
$$

Thus $V_{\alpha}^{1} \subset V_{\alpha}^{2} \subset \cdots$, and $V_{\alpha}^{n}$ is open if $n \geqq 2$; hence, writing $\mathrm{U}_{n} V_{\alpha}^{n}=V_{\alpha}$, we have that $V_{\alpha}$ is open. An easy induction (using (4) and (5)) shows that $\left(V_{\alpha}^{n}, n\right) \subset U_{\alpha}$; hence

$$
V_{\alpha} \subset U_{\alpha}
$$

Further,

$$
U V_{\alpha}=S,
$$

since, given $x \in S$, we have $(x, 1) \in$ some $U_{\alpha}$ (for $V^{1} \Delta$-refines $V$ ), so that, by (3), $x \in V_{\alpha}^{1} \subset V_{\alpha}$.

We also have

(9) Given $x \in V_{\alpha}$, there exists $n>0$ such that $(x, n) \subset V_{\alpha}$.

For there exists $n \geqq 2$ such that $x \in V_{\alpha}^{n-1}$, and then $(x, n) \subset V_{\alpha}^{n} \subset V_{\alpha}$. Next we define, for each $n>0$, a transfinite sequence of closed sets $H_{n \alpha}$, by setting

$$
H_{n 1}=\left(V_{1},-n\right), \quad H_{n \alpha}=\left(V_{\alpha}-\bigcup_{\beta<\alpha} H_{n \beta},-n\right) .
$$

Then we have:

$$
\text { If } \alpha \neq \gamma \text {, no } U^{n} \in U^{n} \text { can meet both } H_{n \alpha} \text { and } H_{n \gamma} \text {. }
$$

For we can suppose $\gamma<\alpha$. Then if $U^{n}$ meets $H_{n \alpha}$, let $x \in U^{n} \cap H_{n \alpha}$; from (3) and (10), $U^{n} \subset V_{\alpha}-\bigcup_{\beta<\alpha} H_{n \beta}$, and so is disjoint from $H_{n \gamma}$. 


$$
\bigcup_{n, \alpha} H_{n \alpha}=S .
$$

For, given $x \in S,(8)$ shows that there will be a first ordinal $\alpha$ for which $x \in V_{\alpha}$; and from (9) there exists $n>0$ such that $(x, n) \subset V_{\alpha}$. We assert $x \in H_{n \alpha}$. For suppose not. Then, from (10) and (3), $(x, n)$ contains a point $y$ not in $V_{\alpha}-U_{\beta<\alpha} H_{n \beta}$; and it follows that $y \in H_{n \beta}$ for some $\beta<\alpha$. But then $x \in\left(H_{n \beta}, n\right) \subset\left(\left(V_{\beta},-n\right), n\right) \subset V_{\beta}$ (from (4)); and this contradicts the definition of $\alpha$.

Write

$$
E_{n \alpha}=\left(H_{n \alpha}, n+3\right), \quad G_{n \alpha}=\left(H_{n \alpha}, n+2\right) .
$$

Thus $H_{n \alpha} \subset E_{n \alpha} \subset \overline{E_{n \alpha}} \subset G_{n \alpha}$, and, as is easily seen from (11),

$$
\text { If } \gamma \neq \alpha \text {, no } U^{n+2} \in U^{n+2} \text { can meet both } G_{n \alpha} \text { and } G_{n \gamma} \text {. }
$$

Write $F_{n}=\mathrm{U}_{\alpha} \overline{E_{n \alpha}}$. Then $F_{n}$ is closed. For suppose $x \in \bar{F}_{n}$. Then every open neighbourhood $N(x)$ of $x$ meets some $\overline{E_{n \alpha}}$ and so meets some $E_{n \alpha}$; but if $N(x)$ is contained in the neighbourhood $(x, n+2)$ of $x$, $N(x)$ can meet at most one set $E_{n \alpha}$ (from (14)), so that $x \in \overline{E_{n \alpha}} \subset F_{n}$.

Finally we define

$$
W_{1 \alpha}=G_{1 \alpha}, \quad W_{n \alpha}=G_{n \alpha}-\left(F_{1} \cup F_{2} \cup \ldots \cup F_{n-1}\right) \quad(n>1) ;
$$

thus the sets $W_{n \alpha}$ are open. We shall show that they form the desired refinement.

In the first place, $\cup_{n, \alpha} W_{n \alpha}=S$. For, given $x \in S$, we have $x \in$ some $H_{n \alpha}$ (from (12)) $\subset \overline{E_{n \alpha}}$; let $m$ be the smallest integer for which there exists $\overline{E_{m \beta}} \ni x$. Then $x \in G_{m \beta}$, and $x \notin F_{1} \cup \cdots \cup F_{m-1}$, so that $x \in W_{m \beta}$.

Next, $W_{n \alpha} \subset G_{n \alpha} \subset\left(H_{n \alpha}, n\right) \subset\left(\left(V_{\alpha},-n\right), n\right) \subset V_{\alpha} \subset U_{\alpha}$ (using (4) and (7)). Thus the sets $W_{n \alpha}$ form an open covering $W$ of $S$ which refines $U$. All that remains to be proved is that $W$ is "locally finite." Given $x \in S$, we have as before that $x \in$ some $H_{n \alpha}$, so $(x, n+3) \subset E_{n \alpha}$ $\subset F_{n}$, and so is certainly disjoint from $W_{k \beta}$ if $k>n$. Further, for a given $k \leqq n$, we have $(x, n+3) \subset U^{n+2} \subset U^{k+2}$, so (13) shows that $(x, n+3)$ can meet $W_{k \beta}$ for at most one value of $\beta$. Thus the neighbourhood $(x, n+3)$ of $x$ meets at most $n$ of the sets $W_{k \beta}$; and the proof is complete.

REMARK. The locally finite refinement $\mathcal{W}$ thus constructed has the additional property that it consists of a countable number of families of sets (formed by the sets $W_{n \alpha}, n$ fixed), the sets of each family having pairwise disjoint closures.

CoROLlaRY 1. Every metric space is paracompact.

For a metric space is fully normal $[5$, p. 53].

CoRollary 2. The topological product of a metric space and a com- 
pact (=bicompact) Hausdorff space is paracompact, and therefore normal..$^{2}$

This follows from Theorems 5 and 1 of [2].

THEOREM 2. Every paracompact space is fully normal (and $T_{1}$ ).

Let $S$ be a paracompact space, and let $U=\left\{U_{\alpha}\right\}$ be a given locally finite open covering of $S$. It will evidently suffice to prove that $V$ has a $\Delta$-refinement.

Open sets $X_{\alpha}$ exist, for each $\alpha$, such that $\bar{X}_{\alpha} \subset U_{\alpha}$ and $U X_{\alpha}=S$. (This follows by an easy transfinite induction argument from the fact that $S$ is normal; cf. [2, Theorems 1 and 6].) By hypothesis, each $x \in S$ has an open neighbourhood $V(x)$ meeting $U_{\alpha}$ only for a finite set of $\alpha$ 's, say for $\alpha \in A(x)$. Let $B(x)$ be the set of those $\alpha$ 's $\in A(x)$ for which $x \in U_{\alpha}$, and let $C(x)$ be the set of $\alpha$ 's $\in A(x)$ for which $x \in \overline{X_{\alpha}}$; clearly $B(x) \cup C(x)=A(x)$. Define

$$
W(x)=V(x) \cap \cap\left\{U_{\alpha} \mid \alpha \in B(x)\right\} \cap \cap\left\{\left(S-\bar{X}_{\alpha}\right) \mid \alpha \in C(x)\right\} .
$$

Evidently $W(x)$ is an open set containg $x$ : hence the sets $\{W(x) \mid x \in S\}$ form an open covering $\mathscr{W}$ of $S$. To verify that $\mathscr{W}$ is a $\Delta$-refinement of $U$, let $y \in S$ be given. There exists a set $X_{\beta} \ni y$; we shall show that the $\operatorname{star}(y, W) \subset U_{\beta}$ that is, that if $y \in W(x)$ then $W(x) \subset U_{\beta}$. For if $y \in W(x)$ then $W(x)$ meets $\bar{X}_{\beta}$ and so clearly $\beta \in A(x)$ and $\beta \notin C(x)$. Thus $\beta \in B(x)$, which implies $W(x) \subset U_{\beta}$, by construction.

Now let $N$ denote the space of positive integers-a countable discrete set-and consider the product $T=\coprod N_{\lambda}(\lambda \in \Lambda)$ of uncountably many copies of $N$. More precisely, the points of $T$ are the mappings $x=\left\{\xi_{\lambda}\right\}$ of the uncountable set $\Lambda$ in $N$ (each $\lambda \in \Lambda$ being mapped on the integer $\left.\xi_{\lambda} \in N\right)$, and a typical basic neighbourhood $U$ of $x$ in $T$ is obtained by choosing a finite set $R(U) \subset \Lambda$ and defining $U$ to consist of all points $y=\left\{\eta_{\lambda}\right\}$ such that $\eta_{\lambda}=\xi_{\lambda}$ for all $\lambda \in R(U)$. $R(U)$ will be called the "set of coordinates restricted in $U . "$

\section{THEOREM 3. The space $T$ is not normal.}

For each positive integer $k$, let $A^{k}$ be the set of all points $x=\left\{\xi_{\lambda}\right\}$ $\in T$ satisfying: for each positive integer $n$ other than $k$, there is at most one $\lambda$ for which $\xi_{\lambda}=n$.

${ }^{2}$ It can be shown that the topological product of a metric space and a normal countably compact space is normal, though not necessarily paracompact. (A space is "countably compact" if every infinite subset has a limit point in the space; cf. $[5$, p. 42$]$. For metric spaces this is equivalent to compactness.) 
It is easy to see that the sets $A^{k}$ are closed and pairwise disjoint. Hence, if $T$ were normal, there would exist disjoint open sets $U, V$ such that $U \supset A^{1}, V \supset A^{2}$. We shall show that this leads to a contradiction.

We shall define inductively sequences of points $x_{n} \in A^{1}$, of integers $0<m(1)<m(2)<\cdots$, and of elements $\lambda_{n} \in \Lambda$, as follows. Define $x_{1}$ to be the point $\left\{\xi_{\lambda}\right\}$ for which $\xi_{\lambda}=1$ (all $\lambda \in \Lambda$ ). Evidently $x_{1} \in A^{1} \subset U$, so $x$ has a basic neighbourhood $U_{1} \subset U$. Let $R\left(U_{1}\right)$ consist of the $m(1)$ elements $\lambda_{k}(1 \leqq k \leqq m(1))$. When $x_{\alpha}$ and $\lambda_{1}, \lambda_{2} \cdots, \lambda_{m(n)}$ have been defined, in such a way that $x_{n} \in A^{1}$ and $\lambda_{1}, \cdots, \lambda_{m(n)}$ are the coordinates restricted in a basic neighbourhood $U_{n} \subset U$ of $x_{n}$, we define $x_{n+1}$ by: $\xi_{\lambda}=k$ if $\lambda=\lambda_{k}(1 \leqq k \leqq m(n))$, and $\xi_{\lambda}=1$ otherwise. Clearly $x_{n+1} \in A^{1}$, so that $x_{n+1}$ has a basic neighbourhood $U_{n+1} \subset U$; and we can always suppose that $R\left(U_{n+1}\right)$ contains $R\left(U_{n}\right)$ as a proper subset. Let $R\left(U_{n+1}\right)$ have $m(n+1)$ elements, and enumerate the elements of $R\left(U_{n+1}\right)-R\left(U_{n}\right)$ as $\lambda_{m(n)+1}, \cdots, \lambda_{m(n+1)}$. The induction is now complete.

Now define a point $y=\left\{\eta_{\lambda}\right\}$ by: $\eta_{\lambda}=k$ if $\lambda=\lambda_{k}(k=1,2, \cdots$, to $\infty)$ and $\eta_{\lambda}=2$ otherwise. Clearly $y \in A^{2} \subset V$, so $y$ has a basic neighbourhood $V_{0} \subset V$. Since $R\left(V_{0}\right)$ is finite, there exists an $n$ such that $\lambda_{k} \in \Lambda$ $-R\left(V_{0}\right)$ whenever $k>m(n)$. Finally, define $z=\left\{\zeta_{\lambda}\right\}$ by:

$$
\begin{array}{ll}
\zeta_{\lambda}=k & \text { if } \lambda=\lambda_{k} \text { with } k \leqq m(n), \\
\zeta_{\lambda}=1 & \text { if } \lambda=\lambda_{k} \text { with } m(n)<k \leqq m(n+1), \text { and } \\
\zeta_{\lambda}=2 & \text { otherwise. }
\end{array}
$$

We evidently have $z \in U_{n+1} \cap V_{0} \subset U \cap V$, giving the desired contradiction.

COROLlaRy. If a product of nonempty $T_{1}$ spaces is normal, all but at most a countable number of the factor spaces must be countably compact. ${ }^{2}$

For otherwise their product would contain a closed subset homeomorphic with $T$; and a closed subset of a normal space is normal.

THEOREM 4. The following statements about a product of nonempty metric spaces are equivalent.

(i) The product is normal.

(ii) The product is fully normal (or paracompact).

(iii) At most $\aleph_{0}$ of the factor spaces are noncompact.

In fact, (ii) $\rightarrow$ (i) [2, Theorem 1], (i) $\rightarrow$ (iii) (Theorem 3, Corollary), and (iii) $\rightarrow$ (ii) from Theorem 1, Corollary 2, since the compact 
factors have a compact product ${ }^{3}$ and the product of the remaining factors is metrisable. ${ }^{4}$

REMARK. In Theorem 4, the hypothesis that the factor spaces be metric cannot be much weakened. This is shown by an example of R. H. Sorgenfrey (see [4]), in which the product of a paracompact (and thus fully normal) space with itself is not even normal.

\section{BIBLIOGRAPHY}

1. P. Alexandroff and H. Hopf, Topologie I, Berlin, 1935.

2. J. Dieudonné, Une généralisation des espaces compacts, J. Math. Pures Appl. vol. 23 (1944) pp. 65-76.

3. C. Kuratowski, Topologie I, Warsaw 1933.

4. R. H. Sorgenfrey, On the topological product of paracompact spaces, Bull. Amer. Math. Soc. vol. 53 (1947) pp. 631, 632.

5. J. W. Tukey, Convergence and uniformity in general topology, Annals of Mathematics Studies, no. 2, Princeton, 1940.

Trinity College, University of Cambridge

${ }^{3}$ A theorem of Tychonoff; see, for example, [5, p. 75] for a simple proof.

4 See, for example, [3, p. 88].

\section{TRANSITIVITY AND EQUICONTINUITY ${ }^{1}$}

\section{W. H. GOTTSCHALK}

Let $X$ be a metric space with metric $\rho$ and let $G$ be a group of homeomorphisms on $X$. If $x \in X$ and $g \in G$, then $x g$ denotes the image of the point $x$ under the transformation $g$. If $x \in X$ and $F \subset G$, then $x F$ denotes $\bigcup_{o \in F} x g . G$ is said to be algebraically transitive provided that $x G=X$ for some $x \in X$ (and therefore for every $x \in X$ ). $G$ is said to be topologically transitive provided that $(x G)^{*}=X$ for some $x \in X$, where the star denotes the closure operator. $G$ is said to be equicontinuous provided that to each $\epsilon>0$ there corresponds $\delta>0$ such that $x, y \in X$ with $\rho(x, y)<\delta$ implies $\rho(x g, y g)<\epsilon(g \in G)$.

With respect to the following lemma compare [4].2

LEMMA. If $X$ is a complete separable metric space and also a multiplicative group, if the center of $X$ is dense in $X$ and if the function $x y$

Presented to the Society, December 31, 1947; received by the editors November 29, 1947.

1 Prepared under the sponsorship of the Office of Naval Research.

${ }^{2}$ Numbers in brackets refer to the bibliography at the end of the paper. 